\title{
Method of the Year 2019: Single-cell multimodal omics
}

\author{
Multimodal omics measurement offers opportunities for gaining holistic views of cells one by one.
}

n 2013, we selected single-cell sequencing as Method of the Year to highlight the ability to sequence DNA and RNA in individual cells, allowing biological differences between cells to be inferred. Since then, the rapid development in singlecell sequencing has broadened our view of cellular heterogeneity and its implications for cell functions. The continued expansion of sequencing technologies further improves our ability to profile different aspects of a cell, including its genome, transcriptome and epigenome, as well as the spatial organization of these -omes.

More recently, technological advances in the single-cell space have enabled multimodal omics measurements, by which we mean here the ability to simultaneously measure multiple modalities (that is, carry out multimodal studies) in one experiment or to integrate different types of omics datasets from multiple experiments. For its great impact in revealing cell functions, discovering relationships across -omes, and recording dynamic biological events, we have selected single-cell multimodal omics as our Method of the Year 2019.

The initial effort in single-cell technology development concentrated on single-cell RNA sequencing (scRNA-seq), which can dissect transcriptomic heterogeneity and reveal previously unknown cell types or cell states in a given complex tissue. Researchers quickly realized that the combination of scRNA-seq with another biomolecular characterization, such as protein profiling, provides information that is more than the sum of its parts. For example, the use of a non-destructive assay, such as cytometric methods, before sequencing can link surface protein expression levels to cellular transcriptomes, allowing a joint analysis of RNA and protein levels. The use of physically separated cellular fractions offers one way to measure in parallel, for example, cytosolic mRNA and the nuclear genome, enabling a complementary cell genotyping by associating the transcriptome with the genome from the same cells.

Lately, the wave of multimodal measurements has been further propagating beyond the transcriptome-centric view. For example, a recent study reports the integration of in situ $\mathrm{Hi}-\mathrm{C}$ and whole-genome bisulfite sequencing for simultaneously profiling chromosome conformation and DNA methylome in single cells, highlighting the growing ability to profile cell-type-specific multimodal measurements (Nat. Methods 16, 991-993, 2019).

Of course, getting to this point has not been without challenges in both experimental design and data analysis. The data coverage of single-cell multimodal omics in experimental measurements remains sparse and noisy. Developments in biochemistry and molecular assays are needed to improve sensitivity, specificity and robustness. High-throughput assays offer a scalable strategy for processing thousands to millions cells in one experiment, but they are still limited to certain types of omics measurements.

In a Comment, Bing Ren and colleagues discuss recent experimental developments in multimodal measurements that profile one cell at a time or provide the high-throughput readout afforded by droplet platforms or combinatorial DNA barcoding systems.

Single-cell multimodal analysis techniques have also opened unprecedented opportunity for the development of advanced statistical and computational methods. One key question is how to perform cross-modal integration and modeling with the datasets generated from the multimodal measurements. As different data types have characteristic statistical, technical and biological features, sophisticated statistical and computational strategies are needed for holistic integration while controlling confounding factors such as batch effects from different sources. Moreover, the availability of such data also drives development of powerful algorithms for in-depth understanding beyond simply revealing cell identity, such as profiling regulatory networks in development systems.

Sarah Teichmann and Mirjana Efremova present an overview and outlook on these exciting fields in their Comment.

The ability to gather data from more than one modality within the same cell is enabling previously challenging biological questions to be addressed. For example, the simultaneous measurement of gene expression and cell surface proteins allows one to better resolve cell subpopulations that retain subtle transcriptomic differences. Optical reconstruction of chromatin architecture (ORCA) in combination with multiplex RNA labeling enables visualization of DNA folding and gene expression in single cells, thus providing a view of $3 \mathrm{D}$ chromatin remodeling in Drosophila embryos (Nature 568, 49-54, 2019). Moreover, sequencing tools in combination with CRISPR-based editing capacity offer an exciting direction for single-cell lineage tracing in developing systems.

From a biologist's point of view, a Comment by Alexander Schier highlights a variety of exciting applications enabled by multimodal measurements of cellular phenotypes and genotypes, such as the ability to correlate mutations and transcriptomes in CRISPR screens.

We also present a News Feature that surveys the continuing efforts from different labs to incorporate single-cell multimodal omics analysis in their work.

Moreover, we present our Methods to Watch as methods and areas that we think will be of interest for their great potential in answering biological questions.

We hope you enjoy reading our Method of the Year 2019 feature. We wish you a happy 2020 !

Published online: 6 January 2020 https://doi.org/10.1038/s41592-019-0703-5 